VoL. 38 (1988) [457-464]

\title{
STRONGLY RIGHT FBN RINGS
}

\author{
HeAKYUNG LeE
}

\begin{abstract}
The annililator of a finite generated $\beta$-critical module is called a $\beta$-coprimative ideal. A prime ideal $P$ is called $\beta$-prime if the Krull dimension of $R / P$ is $\beta$. This paper is concerned with the relationship between the set of $\beta$-prime ideals and the set of minimal $\beta$-coprimitive ideals over a strongly right $F B N$ ring. It is shown that there exists a one-to-one correspondence between the set of $\beta$-prime ideals and the set on minimal $\beta$ coprimitive ideals over a strongly right $F B N$ ring $R$ for $-1<\beta \leqslant \alpha$, where $\alpha$ is the Krull dimension of $R$.
\end{abstract}

\section{INTRODUCTION}

Jategaonkar has shown in [9] that the coprimitive ideals in an $F B N$ ring are prime. But in general this is not true even for right $F B N$ rings. In this paper, we are interested in the lattice of these coprimitive ideals and in particular the question as to when there exists a unique minimal $\beta$-coprimitive ideal for each $\beta$-prime. This question was considered by Boyle and Feller in [4]. They showed that if $R$ is strongly right $F B N$, then there exists a $1-1$ correspondence between the isomorphism classes of indecomposable injectives and the minimal $\beta$-coprimitive ideals for $-1<\beta \leqslant \alpha$, where $\alpha$ is the Krull dimension of the ring $R$. This paper was motivated by an effort to obtain a converse of Boyle and Feller's Theorem.

A right $F B N$ ring $R$ has the property that given a finitely generated module $M$, $R$ satisfies the descending chain condition on annihilators of subsets of $M$. A module with this property is said to be a $\triangle$-module. For a right noetherian ring $R$ with Krull dimension $\alpha$, the set of all right ideals $H$ of $R$ such that the Krull dimension of $R / H$ is strictly less than $\beta$ forms a topology $M_{\beta}$ for each $\beta,-1<\beta \leqslant \alpha$. A strongly right $F B N$ ring is defined to be a ring such that every $M_{\beta}$-critical module is a $\triangle$-module.

In Section 2, we consider the $\beta$-coprimitive ideals which are the annihilators of finitely generated $\beta$-critical modules. We prove that there exists a $1-1$ correspondence between the $\beta$-prime ideals and the minimal $\beta$-coprimitie ideals over a strongly right $F B N$ ring. We also characterise strongly right $F B N$ rings. From this characterisation we determine a converse of Boyle and Feller's Theorem [4, 3.4].

Throughout this paper $R$ denotes an associative ring with identity. All modules are right unital. If $L$ is a subset of a module $M$, then the annihilator of $L$ in $R$ is

Received 3 March 1988

Copyright Clearance Centre, Inc. Serial-fee code: 0004-9729/88 \$A2.00+0.00. 
$\operatorname{ann}(L)=\{r \in R \mid L r=0\}$. The notation $N \leqslant_{e} M$ means that $N$ is an essential submodule of $M$. The injective hull of $M$ is denoted by $E(M)$.

For a module $M$, the Krull dimension of $M$ will be denoted by $|M|$. The definition of Krull dimension and some related results can be found in [8]. If $U$ is a uniform module that contains a critical submodule $C$ with $|C|=\beta$, then we write $c r|U|=\beta$. If $I$ is an indecomposable injective module with $\mathrm{cr}|I|=\beta$, then $I$ is called $\beta$-indecomposable injective. A module $M$ is said to be $\beta$-smooth if for any nonzero submodule $N$ having Krull dimension, $|N|=\beta$. A module $M$ is said to satisfy the large condition if $|M / N|<\beta$ for all essential submodules $N$ of $M$.

If $M$ is a uniform module, then the assassinator of $M$ in $R$ is denoted by ass $(M)=\{r \in R \mid(\exists 0 \neq N \leqslant M)(N r=0)\}$. If a ring $R$ has Krull dimension, then ass $(M)=\operatorname{ann}(C)$ for some critical submodule $C$ of $M$.

\section{STRONGLY RIGHT $F B N$ RINGS}

Throughout this section we assume that $R$ is a right noetherian ring with Krull dimension $\alpha$. Since $R$ is right noetherian, the set of right ideals, $M_{\beta}=\left\{H_{R} \leqslant R \mid\right.$ $|R / H|<\beta\}$ where $-1<\beta \leqslant \alpha$, forms a topology in the sense of [11]. Using this topology, we can define a torsion theory. A module $M$ is called $M_{\beta}$-torsionfree if $\operatorname{ann}(x) \notin M_{\beta}$ for all nonzero elements $x$ of $M$, and $M_{\beta}$-torsion if ann $(x) \in M_{\beta}$ for all $x$ in $M$. Then a module $M$ is called $M_{\beta}$-critical if $M$ is $M_{\beta}$-torsionfree and if $M / N$ is $M_{\beta}$-torsion for all nonzero submodules $N \leqslant M$. If $M$ is $M_{\beta}$-critical, then $M$ is uniform and $\beta$-smooth.

LemMA 2.1. Let $M$ be a module. Then the following statements are equivalent:

(1) $M$ is $M_{\beta}$-critical;

(2) every submodule of $M$ with Krull dimension is $\beta$-critical;

(3) every finitely generated submodule of $M$ is $\beta$-critical.

$R$ is right bounded if every essential right ideal of $R$ contains a nonzero ideal of $R$. A right noetherian ring $R$ is said to be right fully bounded (right $F B N$ ) if each prime factor ring of $R$ is called an $F B N$ ring. It was shown by Amitsur in [1] that a prime ring which satisfies a polynomial identity is right bounded. Cauchon has shown in [5] that a right noetherian ring $R$ is right $F B N$ if and only if every finitely generated module is a $\triangle$-module.

A right noetherian ring is defined in [4] to be strongly right $F B N$ if every $M_{\beta^{-}}$ critical module is a $\triangle$-module. Since $\triangle$-modules play an important role in the study of strongly right $F B N$ rings, we include the following theorem that summaries some of the known properties of $\triangle$-modules.

THEOREM 2.2. [10]: Let $M$ be $\beta$-smooth. The following statements are equiva- 
lent:

(1) $|R / \operatorname{ann}(M)|$ is $\beta$;

(2) $R / \operatorname{ann}(M)$ is $\beta$-smooth;

(3) $M$ is a $\triangle$-module;

(4) $M$ is finitely annihilated.

If $R$ is a strongly right $F B N$ ring, then it is easy to show that $R$ is right $F B N$ by Theorem 2.2 .

Over an $F B N$ ring, every finitely generated critical module is a uniform prime $\triangle$ module by [5] and [9]. This enables us to show that every $M_{\beta}$-critical module $M$ has a prime annihilator and hence $\operatorname{ann}(M)=\operatorname{ann}(C)$ for every finitely generated $\beta$-critical submodule $C$ of $M$. Since $C$ is a $\triangle$-module, $|R / \operatorname{ann}(M)|=|R / \operatorname{ann}(C)|=|C|=\beta$. Therefore $M$ is a $\triangle$-nodule by Theorem 2.2. This argument shows that an $F B N$ ring is strongly right $F B M$.

In particular a strongly right $F B N$ ring is a class of rings between $F B N$-rings and right $F B N$-rings.

Proposition 2.3. If $R$ is a right noetherian $P I$-ring, then $R$ is strongly right $F B N$.

Proof: It is known [1] that a noetherian $P I$-ring is $F B N$, and Cauchon has shown in [6] that a right noetherian prime $P I$-ring is left noetherian. Also Boyle and Feller have shown in [4] that $R$ is strongly right $F B N$ if and only if $R / P$ is strongly right $F B N$ for all minimal prime ideals $P$. Hence if $P$ is a minimal prime ideal of $R$, then $R / P$ is a right noetherian prime $P I$-ring, and thus $R / P$ is a noetherian $P I$-ring. Therefore $R / P$ is $F B N$. By the above remark, $R / P$ is strongly right $F B N$ for all minimal prime $P$, and hence so is $R$.

For $F B N$ rings the annihilator of a critical module is prime, but this is not true for strongly right $F B N$ rings. For example, if $R=\left[\begin{array}{cc}F & F[x] \\ 0 & F[x]\end{array}\right]$ and $M_{R}=\left[\begin{array}{cc}F & f[x] \\ 0 & 0\end{array}\right]$, then $R$ is a strongly right $F B N$ ring and $M$ is a 1-critical module. However $\operatorname{ann}(M)=$ 0 is not a prime ideal. We introduce the definition of the annihilator of a critical module and examine the relationship between prime ideals and these annihilator ideals over a strongly right $F B N$ ring.

An ideal $D$ is called a $\beta$-coprimitive ideal if $D=\operatorname{ann}(C)$ for some finitely generated $\beta$-critical module. A $\beta$-coprimitive ideal will be called a minimal $\beta$-coprimitive ideal if it is minimal in the collection of $\beta$-coprimitive ideals. A prime ideal is called $\beta$-prime if $|R / P|=\beta$. Every $\beta$-prime is a $\beta$-coprimitive ideal.

The critical socle of a module $M$ is the sum of the critical submodules and is denoted by $S M$. If $U$ is a uniform module with $\operatorname{cr}|U|=\beta$, then every finitely 
generated submodule of $S U$ is critical by $[2,3.1]$. Hence $S U$ is $M_{\beta}$-critical by Lemma 2.1. Therefore if $R$ is strongly right $F B N$, then $S U$ is a $\triangle$-module. This gives the first implication in the following lemma.

LEMMA 2.4. The following statements are equivalent for a right noetherian ring $R$ :

(1) $R$ is strongly right $F B N$,

(2) $S U$ is a $\triangle$-module for all uniform modules $U$,

(3) $S I$ is a $\triangle$ for all indecomposable injectives $I$. Moreover, in this situation, ann $(S I)$ is a minimal coprimitive ideal.

Proof: It is enough to prove that (3) implies (1). Let $M$ be $M_{\beta}$-critical. Since $S E(M)$ is a $\triangle$-module and $M \subseteq S E(M)$, then $M$ is a $\triangle$-module.

Since $S I$ is a $\triangle$-module, ann $(S I)=\bigcap_{i=1}^{n} \operatorname{ann}\left(x_{i}\right)$ for some nonzero elements $x_{i}$ of $S I$. Since $x_{1} R+\ldots+x_{n} R$ is a $\beta$-critical submodule of $M$ by Lemma 2.1 , then $\operatorname{ann}(S I)=\operatorname{ann}\left(x_{1} R+\ldots+x_{n} R\right)$ is a $\beta$-coprimitive ideal. Now suppose that $D$ is a $\beta$ coprimitive ideal contained in $\operatorname{ann}(S I)$. Then $D=\operatorname{ann}(C)$ for some finitely generated $\beta$-critical module $C$ and $(S I) D=0$. By $[4,2.2], E E(C) \simeq E(S I)=I$. Therefore, ann $(S I)=D$ and hence ann $(S I)$ is a minimal $\beta$-coprimitive ideal.

In [3], it is shown that there is a $1-1$ correspondence between the isomorphism classes of $\alpha$-indecomposable injective modules and the minimal $\alpha$-coprimitive ideals. However, in general, this is not true for $\beta<\alpha$. For example, consider the ring $R=$ $\left[\begin{array}{cc}F & A / x A \\ 0 & A\end{array}\right]$, where $A=F\left[x,\left({ }^{\prime}\right)\right][z], F$ is a field with derivation (') as in $[7, \mathrm{p}$. $55]$ and $z$ commutes with $x$. Note that $|R|=2$. If $C_{1}=\left[\begin{array}{cc}F & A / x A \\ 0 & 0\end{array}\right]$, then $C_{1}$ is 1-critical and ann $\left(C_{1}\right)=0$. Hence 0 is a minimal 1-coprimitive ideal of $R$. If $C_{2}=\left[\begin{array}{ll}0 & 0 \\ 0 & A\end{array}\right] /\left[\begin{array}{cc}0 & 0 \\ 0 & z A\end{array}\right]$, then $C_{2}$ is also 1-critical and $E\left(C_{1}\right) \neq E\left(C_{2}\right)$. Since 0 is the only minimal 1-coprimitive icleal, there is no 1-1 correspondence between the isomorphism classes of 1-indecomposable injectives and the minimal 1-coprimitive ideals.

Proposition 2.5. Let $R$ be a strongly right $F B N$ ring.

(1) If $D$ is a minimal $\beta$-coprimitive ideal of $R$, then $D=\operatorname{ann}(S I)$ for a $\beta$-indecomposable injective $I$, where $-1<\beta \leqslant \alpha$.

(2) Every $\beta$-prime ideal contains exactly one minimal $\beta$-coprimitive ideal and every minimal $\beta$-coprimitive ideal is contained in a unique $\beta$-prime ideal, where $-1<\beta \leqslant \alpha$. 
(3) Let $P=\operatorname{ass}(I)$ and $D=\operatorname{ann}(S I)$ for some $\beta$-indecomposable injective $I$. Then $P$ is the maximal $\beta$-coprimitive ideal containing $D$ and is the unique $\beta$-prime ideal containing $D$.

Proof: (1) If $D$ is a minimal $\beta$-coprimitive ideal, then $D=\operatorname{ann}(C)$ for some finitely generated $\beta$-critical module $C$. On the other hand, ann $(S E(C))$, then ann $(S E(C))=\operatorname{ann}(C)=D$ by the minimality of $D$.

(2) Let $P$ by a $\beta$-prime ideal. Then $P=$ ass $(I)$ for some indecomposable injective $I$. Since $R$ is right $F B N$, then $\beta=|R / P|=\mid R /$ ass $(I)|=\operatorname{cr}| I \mid$ by $[8,8.6]$. Hence $I$ is a $\beta$-indecomposable injective, and ann $(S I)$ is a minimal coprimitive ideal contained in $P$ by Lemma 2.4. Let $D=\operatorname{ann}(S I)$. Suppose that $P$ contains a minimal $\beta$ coprimitive ideal $D^{\prime} \neq D$. Then by $(1), D^{\prime}=\operatorname{ann}\left(S I^{\prime}\right)$ for some $\beta$-indecomposable injective $I^{\prime}$. Now $P=\operatorname{ann}(C)$ for some finitely generated critical submodule $C$ of $I$. Since $P$ contains $D^{\prime}$, then $C \cdot D^{\prime}=0$. By $[4,2.2], I^{\prime} \simeq E(C)=I$. Hence $D^{\prime}=\operatorname{ann}\left(S I^{\prime}\right)=\operatorname{ann}(S I)=D$, which is a contradiction. Therefore $P$ contains exactly one ninimal $\beta$-coprimitive ideal, which is ann $(S I)$.

Conversely, let $D$ be a minimal $\beta$-coprimitive ideal. Then, by (1), $D=\operatorname{ann}(S I)$ for some $\beta$-indecomposable injective and thus $D \subseteq$ ass $(I)=P$. Since $R$ is right $F B N$, then $|R / P|=c r|I|=\beta$. Hence $P$ is $\beta$-prime. Suppose that $D$ is contained in a $\beta$-prime ideal $P^{\prime} \neq P$. Then there exists a $\beta$-indecomposable injective $I^{\prime}$ such that $P^{\prime}=$ ass $\left(I^{\prime}\right)$. Note that $I^{\prime} \not ॅ I$ and hence ann $\left(S I^{\prime}\right) \neq D$. Now $P^{\prime}$ contains two minimal $\beta$-coprimitive ideals, namely ann $\left(S I^{\prime}\right)$ and $D$, which is impossible. Therefore $D$ is contained in a unique $\beta$-prine ideal $P$.

(3) Let $L$ be a $\beta$-coprimitive ideal properly containing $P=\operatorname{ass}(I)$. Then $L=$ ann $(C)$ for some finitely generated $\beta$-critical module $C$. Since $R$ is strongly right $F B N, C$ is a $\triangle$-module and hence $|R / L|=\beta$ by Theorem 2.2. Since $R / P$ satisfies the large condition, $\beta=|R / L|<|R / P|=\beta$, which is a contradiction. Therefore $P$ is the maximal $\beta$-coprimitive ideal containing $D$.

By Proposition 2.5(3), the lattice of $\beta$-coprimitive ideals over a strongly right $F B N$ ring has a maximal element and a minimal element. However, the lattice can be more complicated between these elements. The following example shows that the lattice of $\beta$-coprimitive ideals need not be linearly ordered.

Example 2.6. Let $R=\left[\begin{array}{cc}F & F[x] \oplus F[x] \\ 0 & F[x]\end{array}\right]$. Then $R$ is a strongly right $F B N$ ring. Let $L_{1}=\left[\begin{array}{cc}0 & F[x] \oplus 0 \\ 0 & F[x]\end{array}\right]$ and $L_{2}=\left[\begin{array}{cc}0 & 0 \oplus F[x] \\ 0 & F[x]\end{array}\right]$. Then $C_{1}=R / L_{1}$ and $C_{2}=$ $R / L_{2}$ are 1-critical. Hence $D_{1}=\operatorname{ann}\left(C_{1}\right)=\left[\begin{array}{cc}0 & F[x] \oplus 0 \\ 0 & 0\end{array}\right]$ and $D_{2}=\operatorname{ann}\left(C_{2}\right)=$ 
$\left[\begin{array}{cc}0 & 0 \oplus F[x] \\ 0 & 0\end{array}\right]$ are 1-coprinuitive ideals. On the other hand, ass $\left(C_{1}\right)=\operatorname{ass}\left(C_{2}\right)=P=$ $\left[\begin{array}{cc}F & F[x] \oplus F[x] \\ 0 & 0\end{array}\right]$ and $|R / P|=1$. Thus $P$ is 1-prime. Since $R$ is also right $F B N$, then there is an isomorphism $f: E\left(C_{1}\right) \simeq E\left(C_{2}\right)$. Therefore $f\left(C_{1}\right)+C_{2}$ is 1-critical by $[2,3.1]$, and $\operatorname{ann}\left(f\left(C_{1}\right)+C_{2}\right)=D_{1} \quad D_{2}=0$ is a minimal 1-coprinitive ideal. Now we have the following diagram in the lattice of 1-coprimitive ideals of $R$.

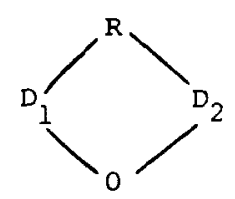

We can now characterise strongly right $F B N$ rings. Further, this provides a situation when the converse of Boyle and Feller's theorem [4, 3.4] holds.

THEOREM 2.7. Let $R$ a ring. Then the following statements are equivalent:

(1) $R$ is a strongly right $F B N$ ring;

(2) $R$ is right $F B N$ and $R$ has the descending chain condition on $\beta$ coprimitive ideals, where $\beta$ is an ordinal with $-1<\beta \leqslant \alpha$,

(3) $R$ is right $F B N$ and $\operatorname{ann}(S I)$ is a minimal $\beta$-coprimitive ideal for all $\beta$-indecomposable injectives, for $-1<\beta \leqslant \alpha$.

(4) $R$ is right $F B N$ and every $\beta$-prime ideal contains a minimal $\beta$-coprimitive ideal for $-1<\beta \leqslant \alpha$;

(5) $R$ is right $F B N$ and the correspondence $\phi: P \rightarrow \operatorname{ann}\left(S I_{p}\right)$, where $I_{p}$ is an indecomposable injective summand of $E(R / P)$, is a bijection between the set of $\beta$-prime ideals and the set of minimal $\beta$-coprimitive ideals for $-1<\beta \leqslant \alpha$;

(6) $\quad R$ is right FBN and the correspondence $\psi: I \rightarrow \operatorname{ann}(S I)$ is a bijection between the isomorphism classes of $\beta$-indecomposable injectives and the set of ninimal $\beta$-coprimitive ideals for $-1<\beta \leqslant \alpha$.

Proof: $(1) \Rightarrow(2)$ Let $D_{1} \supseteq D_{2} \supseteq \ldots$ be a descending chain of $\beta$-coprimitive ideals. Then for each $i, D_{1}=$ ann $\left(C_{i}\right)$ for some finitely generated $\beta$-critical module $C_{i}$. Since $R$ is right $F B N$ and ann $\left(C_{i}\right) \subseteq$ ann $\left(C_{1}\right)$ for all $i \geqslant 1$, then $E\left(C_{1}\right)=E\left(C_{i}\right)$ for all $i \geqslant 1$ by $[4,2.2]$. Hence $\sum_{i=1}^{\infty} C_{i}$ can be considered as a submodule of $S I_{1}$, where $I_{1}=E\left(C_{1}\right)$. Since $R$ is strongly right $F B N$, ann $\left(S I_{1}\right)$ is a minimal $\beta$-coprimitive ideal and is contained in $D_{i}$ for all $i$. Let $D=\operatorname{ann}\left(S I_{1}\right)$ and consider the descending 
chain of $D_{1} / D \supseteq D_{2} / D \supseteq \ldots$ Since $R$ is right $F B N$, then $R / D$ is $\beta$-smooth for all $i$. Thus $\left|D_{i-1} / D_{i}\right|=\beta$ for $i \geqslant 2$. This contradicts to the fact that $|R / D|=\beta$. Therefore the chain is finite.

(2) $\Rightarrow(3)$ Let $I$ be a $\beta$-indecomposable injective, and let $C_{1}$ be a critical submodule of $I$. Then $D_{1}=\operatorname{ann}\left(C_{1}\right)$ is a $\beta$-coprimitive ideal. If $(S I) \cdot D_{1} \neq 0$, then there exists a $\beta$-critical submodule $C_{2}$ of $S I$ such that $D_{2}=\operatorname{ann}\left(C_{2}\right)$ and $D_{1} \nsubseteq D_{2}$. By $[2,3.1], C_{1}+C_{2}$ is a critical submodule of $S I$, and hence $D_{1} \cap D_{2}=$ ann $\left(C_{1}+C_{2}\right)$ is a $\beta$-coprimitive ideal. Continuing in this manner, we form a descending chain of $\beta$-coprimitive ideals $D_{1} \supseteq D_{1} \cap D_{2} \supseteq \ldots$. By hypothesis, this chain must stop with a $\beta$-coprimitive ideal $D$. By the construction, $D=\operatorname{ann}(S I)$. As in the proof of Lemma 2.4 , ann $(S I)$ is a minimal $\beta$-coprimitive ideal.

(3) $\Rightarrow$ (4) Let $P$ be a $\beta$-prime ideal. Then $P=$ ass $(I)$ for some indecomposable injective $I$. Since $R$ is right $F B N$, then $c r|I|=|R / P|$ by $[8,8.6]$. However, $P$ being $\beta$-prime implies that $I$ is a $\beta$-indecomposable injective. Hence by hypothesis, ann $(S I)$ is a minimal $\beta$-coprimitive ideal, and ann $(S I) \subseteq$ ass $(I)=P$.

$(4) \Rightarrow(5)$ Let $P$ be a $\beta$-prime ideal, and let $D$ be a minimal $\beta$-coprimitive ideal contained in $P$. We claim that ann $\left(S I_{p}\right)=D$, where $I_{p}$ is an indecomposable injective summand of $E(R / P)$. Let $D=\operatorname{ann}(C)$ for some finitely generated $\beta$-critical module $C$. Since $R$ is right $F B N$, then $E\left(C^{\prime}\right) \simeq I_{p}$, and thus $D=$ ann $\left(C^{\prime}\right)$ for some $\beta$-critical submodule $C^{\prime}$ of $I_{p}$. If $\left(S I_{p}\right) \cdot D \neq 0$, then there exists a $\beta$-critical submodule $N^{\prime}$ of $I_{p}$ such that $N^{\prime} \cdot D \neq 0$. Let $D^{\prime}=\operatorname{ann}\left(N^{\prime}\right)$. Then by $[2,3.1], C^{\prime}+N^{\prime}$ is a $\beta$-critical submodule of $I_{p}$ and hence $D \cap D^{\prime}=$ ann $\left(C^{\prime}+N^{\prime}\right)$ is a $\beta$-coprimitive ideal contained in $D$. Hence $D=D^{\prime}$ by the minimality of $D$. This is a contradiction. Therefore $D=\operatorname{ann}\left(S I_{p}\right)$. That the indicated map is a bijection now follows from Proposition $2.5(2)$.

$(5) \Rightarrow(6)$ : Since $R$ is right $F B N$, by $[8,8.6]$ there is a $1-1$ correspondence between the isomorphism classes of indecomposable injectives and prime ideals given by $I \rightarrow$ ass $(I)$. Therefore the result follows from (5).

$(6) \Rightarrow(1)$ : Let $I$ be indecomposable injective. Then ann $(S I)$ is a minimal coprimitive ideal. Since $R$ is right $R B N$, then $|R / \operatorname{ann}(S I)|=c r|S I|$. Therefore $S I$ is a $\triangle$-module by Theorem 2.2. Hence by Lemma $2.4, R$ is strongly right $F B N$.

COROLlARY 2.8. If $R$ is right $F B N$, then $R$ is strongly right $F B N$ if and only if there is a 1-1 correspondence between the isomorphism classes of indecomposable injectives and the minimal $\beta$-coprimitive ideals for $-1<\beta \leqslant \alpha$, given by $I \rightarrow \operatorname{ann}(S I)$.

\section{REFERENCES}

[1] S.A. Amitsur, 'Prime rings having polynomial identities with arbitrary coefficients', Proc. London Math. Soc. (3) 17 (1967), 470-486. 
[2] A.K. Boyle and E.H. Feller, Semicritical modules and $\beta$-primitive rings, in module theory, Lecture Notes in Math. 700 (Springer-Verlag, New York, Heidelberg, Berlin, 1979).

[3] A.K. Boyle and E.H. Feller, ' $\alpha$-coprimitive ideals and $\alpha$-indecomposable injective modules', Comm. Algebra 8 (1980), 1151-1167.

[4] A.K. Boyle and E.H. Feller, ' $\triangle$-socles and coprimitive ideals', Revue Roumaine De Mathematiques Pure et Appliquees 31 (1986), 189-197.

[5] G. Cauchon, 'Les $T$-anneaux, la condition $(H)$ de Gabriel et ses consequences', Comm. Algebra 4 (1976), 11-50.

[6] G. Cauchon, 'Anneaux semi-premiers, Noethériens, à identités polynômiales', Bull. Soc. Math. France 104 (1976), 99-111.

[7] N.J. Divinsky, Rings and radicals, Mathematical exposition 14, (University of Toronto Press, 1964).

[8] R. Gordon and J.C. Robson, 'Krull dimension', Mernoirs Amer. Math. Soc. 133 (1973).

[8] A.V. Jategaonkar, 'Jacobson's conjecture and modules over fully bounded noetherian rings', $J$. Algebra 30 (1974), 103-112.

[10] C. Nastasescu, 'Modules $\triangle$-injectifs sur les anneaux à dimension de Krull', Comm. Algebra $\theta(13)$ (1981), 1395-1426.

[11] B. Stenstrom, Rings of quotients (Springer-Verlag, Berlin and New York, 1972).

Department of Mathematics

Winthrop College

Rock Hill, SC. 29733

United States of America. 